\title{
Could it be a small world after all?
}

\section{Sophisticated technologies can now explore nano-scale forces and interactions. But most biologists are staying on the sidelines, waiting to see if these technologies can really help them. Nathan Blow reports.}

For years, biophysicists have been characterizing the small forces associated with cells and molecules using a variety of techniques born in the worlds of physics, chemistry and even materials science. They are pushing their instruments further in the attempt to measure molecular forces in the sub-piconewton range, and some biologists, at least, are becoming more interested in finding out the extent to which these forces have a role in biological processes.

"The more that the biological community thinks about the role of forces, the more likely it will be that these measurements are made," says Daniel Fletcher, a bioengineer at the University of California, Berkeley. But he adds that it is a real "chicken and egg" situation: how can you convince yourself that forces are important if you do not measure them, but if they are not important, why measure them?

"We need other biologists to use these techniques," says Carlos Bustamante, a biophysicist at the University of California, Berkeley. Bustamante has been developing and using 'optical traps' (which hold and manipulate molecules by means of small forces) for more than a decade as part of his work investigating the effects of very small forces on biomolecules. He thinks that if more biologists started to use these tools, the technology would move further, faster. There is consensus on one thing: techniques and instrumentation will have to become more robust and user-friendly if any wider adoption of these methods is going to take place.

One emerging trend that might pique the interest of biologists hesitant to explore the nanoscale world is the integration of different technologies. Whether it is combining Foerster resonance energy transfer (FRET) with optical traps or carbon nanotubes with atomic force microscopy (AFM) or even AFM with confocal microscopy, different tools are being integrated in an attempt to maximize our understanding of highly complex molecular interactions, while potentially providing novel insights into the world of the single molecule.

\section{Microscopy by force}

AFM, which was developed more than 20 years ago, has a resolution of a fraction of a nanometre $-1,000$ times better than the optical diffraction limit. This resolution is achieved by scanning a sample with a cantilevered tip and measuring deflection of the cantilever with a laser to give topological information such as height, length and shape. Different in principle from optical microscopy, AFM's 'touch scanning' approach can image a surface and measure its associated forces at the same time.
AFM was first used in materials science and physics to examine surface properties of semiconductors for process-control applications. And although the interest of biophysicists in applying AFM to study cell surfaces and interactions between single molecules has resulted in advances in the technology, including new modes of scanning in liquids and with live cells, AFM is still a baby in the biology world. "It is relatively young in its development, but I see more and more cell biologists starting to explore the potential of AFM and similar methods to investigate the role and influence of mechanical properties," says Sebastian Tille, senior life sciences product marketing manager at Veeco Instruments in Woodbury, New York.

\section{Hooke's law}

The basis of AFM lies in Hooke's law of elasticity - a simple equation expressing the force generated within a spring when compressed in terms of a 'spring constant' and the length of the spring. Developers have been toying with this relationship for years in their efforts to improve the springs (or cantilevers) used in AFM.

"Cantilevers are essentially springs, typically 50-200 micrometres in length," says Roger Proksch, president of AFM specialists Asylum Research in Santa Barbara, California, "and when they are manufactured, there is a degree of uncertainty in their dimension." As the spring constant depends on these dimensions, this uncertainty can lead to measurement

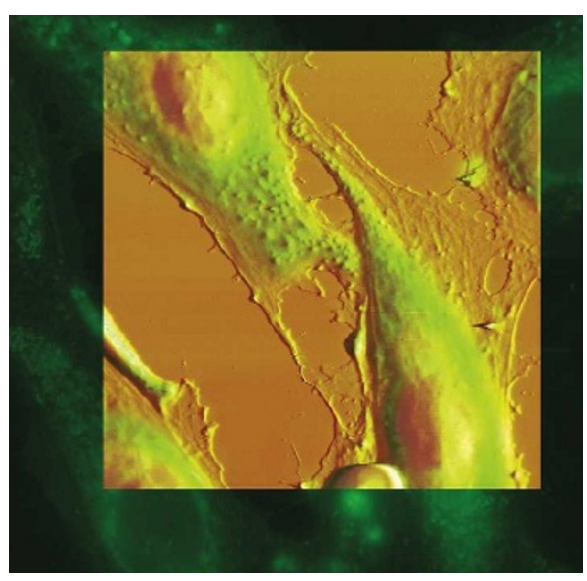

Fluorescence image of live cells overlayed on an atomic force microscopy topographic image of the same cells.
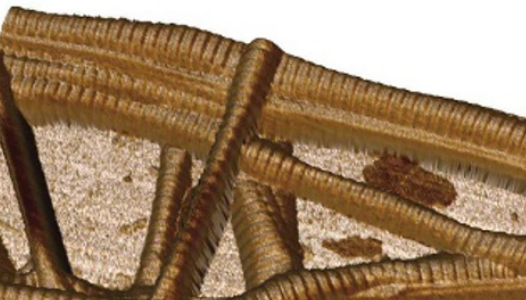


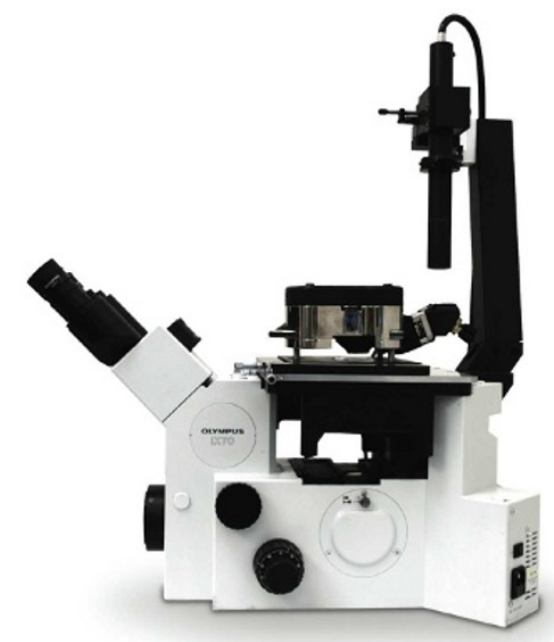

Asylum Research's MFP-3D AFM system can be combined with different microscopy modes.

a simple analogy: imagine twanging a ruler hanging off the end of a desk, it vibrates at a certain frequency. Now if the ruler were shrunk by a factor of 1,000 , the frequency of vibration would dramatically increase. "Today a cantilever in fluid has a resonance frequency of 5-50 kilohertz, we would like to see cantilevers that go from $500 \mathrm{kHz}$ to $5 \mathrm{MHz}$," he says, noting that a smaller cantilever is something he expects to see in the very near future.

Length or, in the case of AFM, the distance from the cantilever tip to the surface, is the other part of Hooke's law and an issue that developers have worked hard on, notably by using piezoelectric materials to move AFM tips. "Piezo's are great: turn a knob, change the potential by a volt and you can move an ångstrom," says Proksch, who also notes that implementing the idea was no small feat. When the potential across the piezoelectric material is changed it will move an ångstrom as desired, but then in the next few minutes, or even seconds, it will move another ångstrom - a phenomenon known as creep. Researchers have attacked this problem and a related problem - hysteresis - in a number of ways, with some AFM developers, including Asylum, deciding to place sensors around the piezoelectric material to measure the creep directly. Fletcher says that with these sensors providing feedback as the tip moves over the surface, this form of drift has been largely eliminated - although his group has had to work through drift issues of its own (see 'When one cantilever is not enough').

Many of the advances that AFM developers are working on now involve moving the technology into the hands of biologists. Both Veeco and Asylum have started to combine optical and force microscopes in a single system. Tille notes that Veeco's BioScope II is compatible with advanced light microscopy modalities when the AFM is placed on the top of an inverted microscope.
"With registration between the optical image and the AFM tip, the user can select regions of interest to correlate fluorescence signals in cells with high-resolution topography," Tille says. In addition to Asylum and Veeco, other companies such as Park Systems in Santa Clara, California, and Novascan Technologies in Ames, Iowa, are exploring combining various optical microscopy techniques with AFM.

\section{Feeling single-molecule interactions}

"I think one of the most important advantages of AFM is that you can measure a structure's dynamics and function at the same time," says Peter Hinterdorfer, a biophysicist at the Institute for Biophysics at Johannes Kepler University in Linz, Austria.

Hinterdorfer's group has been exploring the molecular recognition of ligands and receptors by combining AFM with antibodies to simultaneously obtain cell topography and recognition images in a method the group calls TREC. "This is basically a counterpart to immunofluoresence in which we try to localize receptors," explains Hinterdorfer. The AFM tip is functionalized by attaching antibodies and then is brought into contact with the cell membrane. The binding between ligand and receptor can be measured, while obtaining topographical information at the same time.

Hinterdorfer is quick to point out that the advantage of AFM for TREC, compared with other optical techniques, is its higher lateral resolution, which can be combined with the ability to obtain mechanical parameters of the cell surface. Although his group developed TREC several years ago, most of the work has been done with either model systems with membrane preparations or fixed cells. "For single cells there is a lot more to do, because at the moment we are used to TREC on fixed cells, but we are working on live cells as well," says Hinterdorfer, adding that as high-speed AFM is improved, he expects to see more work following physiological processes in living cells using methods such as TREC.

Whereas Hinterdorfer and others use AFM to measure interactions between individual molecules, other groups are trying to visualize such interactions. Some groups are investigating carbon nanotubes as sensors. "Our interest in carbon nanotubes for biological applications is mainly as optical

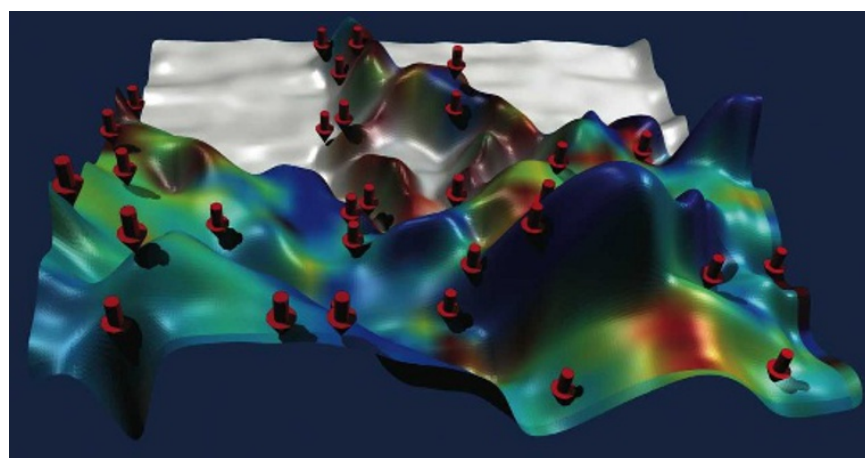

Interaction map of the surface of a HeLa cell using functionalized AFM.

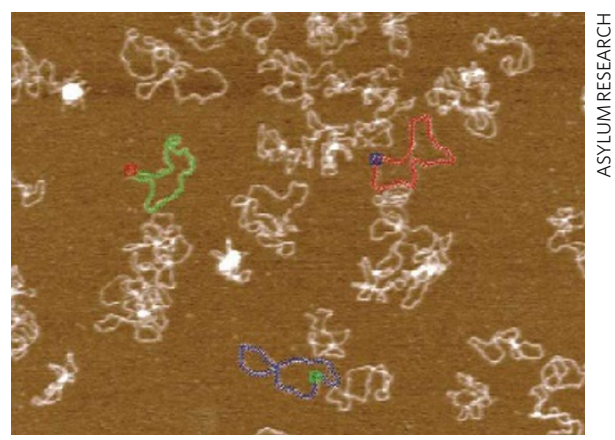

AFM software offers the ability to outline the contours of individual molecules.

diagnostics," says Michael Strano, a chemical engineer at the Massachusetts Institute of Technology in Cambridge. "Carbon nanotubes can tell you about the chemical environment of a very small region of space," he says, and they have optical properties that make them unique for sensor applications (see 'Shrinking down gas chromatography').

Carbon nanotubes can emit light in the near infrared, which eliminates issues of background fluorescence as this is a part of the spectrum in which very few biological systems autofluoresce. In addition, unlike dyes, or even quantum dots, which can photobleach over time, carbon nanotubes are extremely photostable, allowing measurement of fluorescence changes to obtain molecular-level information.

Strano and his group have spent a lot of time working out how to get carbon nanotubes into living cells and to understand how and why, once inside, they move around. Using singleparticle tracking methods, they recently showed that the nanotubes can either diffuse around the cell in a confined pattern or be actively transported. "We are systematically studying these complex behaviours," says Strano, "it is going to take some effort to sort out and ultimately learn to control them."

Strano's team and others are interested in the application of carbon nanotubes as optical sensors to detect single-molecule binding events. "Our lab has recently demonstrated that we can detect biologically important molecules binding to nanotubes at the single-molecule level in real time," says Strano. The one-dimensional electronic property of carbon nanotubes means high electron density at a very narrow region of energy. One result of this property is that when a carbon nanotube absorbs a particular photon, the absorbance has a very sharp maximum. This allows a single carbon nanotube to be visualized with spectroscopy, and also gives researchers the opportunity to see if a nanotube responds to a biomolecule in a single-molecule association event.

"We have few tools to study analyte fluxes at very low concentration levels," notes Strano. His idea is to use carbon nanotubes as local amplifiers of a single interaction 
event, because the event disproportionately affects the nanotube properties and the nanotube has such a large optical cross-section. Other groups are exploring different technologies to detect single-molecule interactions.

Taekjip Ha is a physicist from the University of Illinois at Urbana-Champaign who has been developing fluorescence resonance energy transfer (FRET) approaches for studying single-molecule interactions. "It took several years to convince ourselves that this technique was going to be very useful to obtain biophysical and biological measurements," says $\mathrm{Ha}$.

Although some studies using single-molecule FRET have been done in living cells, the vast majority of experiments have been in vitro. "This is due to technical limitations," says Ha, "the probes are not bright enough for robust measurements in living cells." But he does think that the use of quantum dots could enable the more widespread use of single-molecule FRET in living cells - with one small change. "It is currently difficult to do a single-molecule
FRET experiment using quantum dots because those made commercially are too big."

A quantum dot plus its coating, which is needed to solubilize the dot and conjugate to a biomolecule, can easily be more than 20 nanometres in diameter - much larger than the 10 nanometres maximum separation required for a FRET experiment. Ha thinks that single-molecule FRET in living cells will have to wait until the next generation of quantum dots. He thinks this is only a matter of time, as he sees no reason why quantum dots cannot be made smaller.

\section{Setting the trap}

"The area of single-molecule manipulation goes back to 1991," recalls Bustamante. "At that time we did an experiment using gravity and the weight of little beads to extend the DNA." Bustamante and others soon turned their attention from beads and gravity to a more sophisticated technique called 'optical tweezers', developed by Stephen Chu, currently at University of California, Berkeley, and Arthur Ashkin, now retired from Bell Labs and Lucent Technologies. This technique can trap and manipulate molecules with diameters of nanometres.

Optical tweezers make use of a focused laser beam to trap and hold dielectric objects. Developed in the late 1980s and used extensively in the 1990s, researchers only recently started to see a need for even higher-resolution optical tweezer systems. "I think during that earlier time our objective was to be able to follow and extend molecular motors, see molecules unfold and measure the mechanical properties of nucleic acids," says Bustamante, "and you could do quite a bit of research without going into extremely high resolution." But today, Bustamante thinks the biology has actually pulled biophysicists towards studying things at a smaller scale - requiring more stability, less drift, more spatial resolution and improved temporal resolution from optical tweezers.

For high-resolution optical tweezers to work, the key issue of drift had to be overcome. One approach, developed by Steven Block's group

\section{WHEN ONE CANTILEVER IS NOT ENOUGH}

"I did my doctoral work building force and optical microscopy systems," says Daniel Fletcher, a bioengineer at the University of California, Berkeley. "I was an instrument developer and did not really get to use them, as the main goal was always to develop a new contrast mechanism or achieve the best resolution." But all that changed when Fletcher started his postdoc training and saw cells crawling.

"I realized that although we could build these fancy instruments that control forces down to the piconewton level or below, we had no clue how to assemble complex molecular systems that do what the crawling cells could do." This led Fletcher to study the dynamic actin networks of crawling cells using atomic force microscopy (AFM) and optical traps.

The biochemistry of the actin system has been studied for more than 20 years and actin networks can be grown in vitro, which provides the opportunity to directly probe how network assembly is involved in force generation and shape-changes in cells. "What we have been trying to do with both AFM and optical traps is to create a controlled resistance to

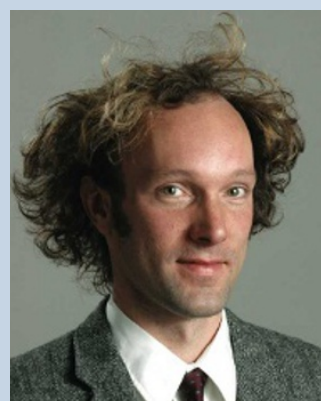

Daniel Fletcher studies actin networks using atomic force microscopy. the growth of these networks and study their behaviour," he says. One approach that Fletcher's group uses is to grow actin networks on the ends of its AFM cantilever and then monitor how the networks adapt to different loads and how those forces influence the architecture of the structures generated.

Using AFM in this manner provided a challenge, requiring Fletcher to use his instrumentdevelopment skills. "One of the things that AFM is not good at doing is measuring sustained displacement over long time periods, because the surface can drift relative to the cantilever,"

\section{says Fletcher.}

Cantilevers are not directly connected to the surface being scanned - they are held by a fluid cell, held by a mount, which is connected to a moving stage that is connected through an even longer path to the surface. The distance between cantilever and surface helps to make crucial calculations of the extent of deflection of the cantilever by the surface. Fletcher explains that because of this large mechanical path from the surface to the cantilever, which can alter the reference position of the cantilever relative to the surface due to thermal drift, the forces measured over time can change even if the sample does not. At short time scales this might not be an issue, but for his team, trying to measure actin growth continuously on the microscope tip over hours, drift of this sort can potentially influence the data collected.

Fletcher decided that one approach to correcting for this drift would be a dual cantilever
AFM system. Having two adjacent cantilevers allows one cantilever to always be in contact with the surface while the second measures the growth of the actin networks. Fletcher says that by always having one cantilever on the surface, they can now detect movement or drift directly and use feedback to correct the cantilever's position.

Fletcher continues to develop new approaches to making force measurements. His team recently developed an epi-fluorescence 'side-view' AFM device (most commercial systems come with the AFM system mounted on top of a microscope) to visualize growing actin networks between the cantilever and the surface. "Where people interested in single-, or even multimolecule, biophysics can continue to make contributions is in developing better tools that help to overcome the limitations of existing technologies and reveal new behaviour of biological systems."

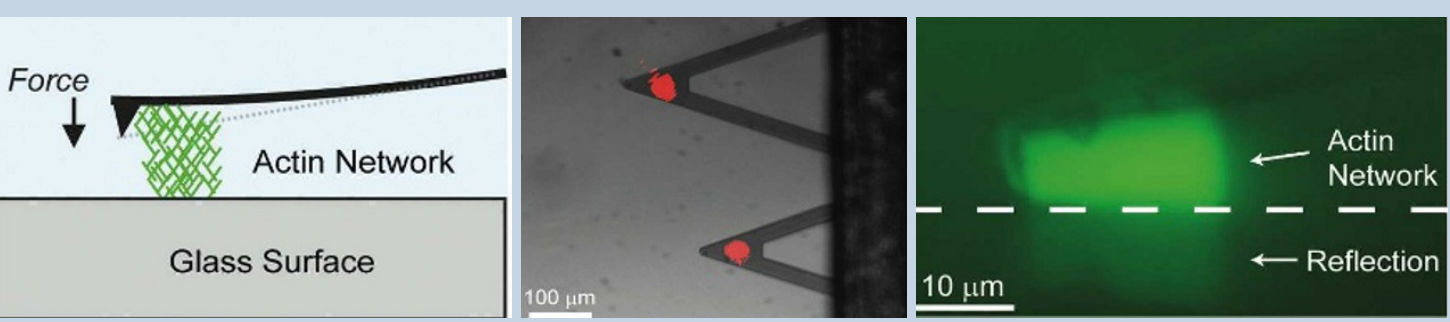

Side-view AFM (left) and the two-cantilever approach (centre) for studying actin-network growth. 
at Stanford University, California, is to attach a molecule to two beads and use two optical tweezers, neither of which is physically attached to the lab environment, to hold the molecule. Bustamante's group recently added another element to this approach - the use of a single laser beam that splits into the two optical tweezers, to control for variation in laser light by providing feedback on both traps (J. R. Moffitt et al. Proc. Natl Acad. Sci. USA 103, 9006-9011; 2006).

Companies are eyeing the area of optical tweezers seriously. Carl Zeiss in Jena, Germany now offers the Palm Micro Tweezers that use either a red or near infrared laser that can be split into two independent traps. Arryx, in Chicago, Illinois, has taken a different approach for its optical-tweezer technology, called BioRyx 200, which is a holographic optical trap created from a spatial light modulator and allows the control of up to 200 objects simultaneously.

$\mathrm{Ha}$ is starting to combine optical tweezers with single-molecule FRET to study the enzymes involved in transcription and translation.

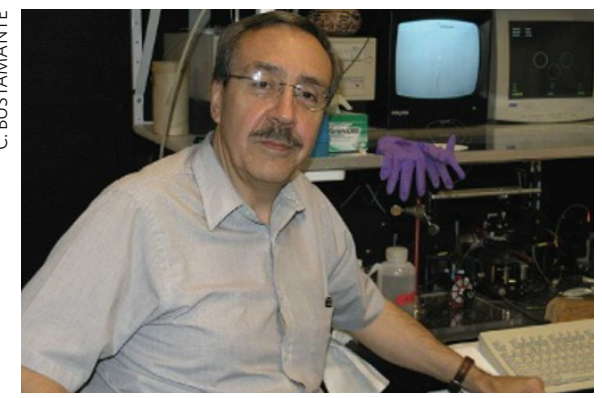

Carlos Bustamante would like to see a wider adoption of techniques to study single molecules.
He thinks that the combination of these techniques is necessary as single-molecule research moves towards studying more complex properties of enzymes and proteins. "It is now possible to measure the fundamental reaction steps of the enzymes using optical traps to obtain single base pair resolution," says $\mathrm{Ha}$, but he adds that using FRET allows conformational changes of the enzymes themselves to be monitored at the same time. This combination allowed Ha's team to directly measure via fluoresence conformational changes as a function of applied force to dissect the landscape of the Holliday junction - a mobile junction between four strands of DNA (S. T. Hohng et al. Science 318, 279-283; 2007).

\section{Added colour}

$\mathrm{Ha}$ is moving into three-colour FRET with optical traps to get more information from each experiment. Whereas two-colour FRET can measure one distance, multiple-colour FRET will provide additional distance information from each experiment. Ha is using three-colour FRET (one donor and two acceptors) to study the diffusion of protein on single-stranded DNA and the enzymatic activity of the ribosome.

Bustamante is also excited about the possibility of using FRET with optical tweezers. "The bottom line is that it will allow you to follow a particular biological process in a multidimensional fashion,"he says. His lab has built a prototype optical trap, called 'minitweezers', that will incorporate fluorescence. With this, the team hopes to bring together the whole optical table into an instrument the size of two

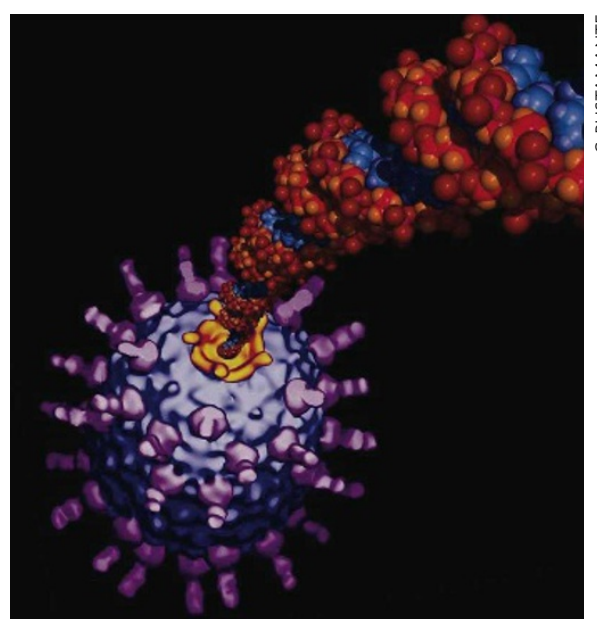

Schematic representation of a molecule attached to a bead within an optical trap.

\section{coffee cans, Bustamante says.}

Measurements of force and interaction at the single-molecule level are being reported with increased frequency. And there is interest from the wider biological community: at the 2007 meeting of the American Society for Cell Biology there was a session focused on the analysis of mechanical forces. "It was pretty amazing to see a ballroom of more than a thousand people listening to talks on the significance of mechanical properties of cells," Tille says. And with developers actively trying to engage biologists while continuing to advance the power of these technologies, one has to wonder how many more will be listening next year. Nathan Blow is technology editor for Nature and Nature Methods.

\section{SHRINKING DOWN GAS CHROMATOGRAPHY}

Imagine looking down at your watch to check the time, while the same watch performs chromatography to determine the chemical composition of the air around you. Although unlikely to be in the shops any time soon, recent advances in carbon nanotube technology and surface chemistry could make very small gas-chromatography devices a future reality.

Michael Strano's group at the Massachusetts Institute of Technology in Cambridge has been developing a highly sensitive, miniaturized gaschromatography instrument based on carbon nanotubes. Sensitivity was key for this device, Strano wanted to be able to detect analytes in the part-per-trillion range - an amount so small it can be difficult to grasp at first. "This is like opening a small vial in a crowded high-school gymnasium,"
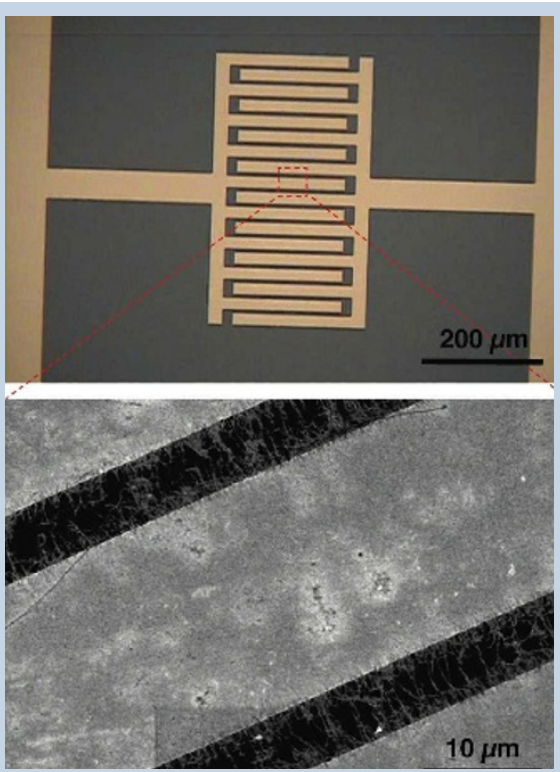

Micro-GC nanotube sensor array (above); close up of a nanotube with array (below).

explains Strano, "even if you open the vial and shut it quickly, you have actually released hundreds of parts per trillion of this mixture everywhere."

For the backbone of its new gas-chromatography instrument, Strano's group created singlewalled nanotube, chemi-resistor arrays in which the nanotubes were aligned between electrodes.

Changes in the electrical resistance can be analysed as a desired molecule binds the nanotube. To tune the binding of the analyte to the arrays the group also had to develop a new chemistry (C. Y. Lee and M. S. Strano J. Am. Chem. Soc. 130, 1766-1773;

2008). "You can use mean basicity to target whether your molecules will bind very strongly, in between, or not at all," explains Strano. Then using a microelectromechanical systems device it should be possible to have precise control over which molecules bind to the array.

As a proof-of-principle, Strano's group created a very simple device by etching a chromatography column on a chip. Using a 100micrometre trench the team showed that mixtures can be separated and, using a fast detector, it is possible to transduce each peak as it comes out.

Strano group's demonstrated parts-per-trillion detection on its device, confirming the potential of this approach to miniaturized, onchip gas chromatography. And for Strano, seeing an actual physical device working at that level was extremely rewarding. “We are engineers, so we try to make devices that work." 